PHYSICA

\title{
Econophysics: Can physicists contribute to the science of economics?
}

\author{
H.E. Stanley ${ }^{\mathrm{a}, *}$, L.A.N. Amaral ${ }^{\mathrm{a}}$, D. Canning ${ }^{\mathrm{b}}$, \\ P. Gopikrishnan ${ }^{\mathrm{a}}$, Y. Lee ${ }^{\mathrm{a}}, \mathrm{Y}$. Liu ${ }^{\mathrm{a}}$ \\ ${ }^{a}$ Center for Polymer Studies, Department of Physics, Boston University, Boston, MA 02215, USA \\ ${ }^{\mathrm{b}}$ Harvard Institute for International Development, Harvard University, Cambridge, MA 02138, USA
}

Received 3 May 1999

\begin{abstract}
Econophysics is a new word, used to describe work being done by physicists in which financial and economic systems are treated as complex systems. Everyone is affected by economic fluctuations, and quantifying fluctuations is a topic that many physicists have contributed to in recent years. Moreover, everyone - rich and poor - would be powerfully affected by a breakdown of the world-wide financial system. Further, it is possible that methods and concepts developed in the study of strongly fluctuation systems might yield new results in economics. Finally, economic systems are complex interacting systems for which a tremendous amount of quantitative data exists, much of it never analyzed. Here we discuss selected recent examples where statistical physicists studying fluctuations have uncovered two new empirical "laws". The first empirical law concerns the histogram giving the relative occurrence probability that a stock experiences a given price change; this histogram decreases as the given price change increases, with an apparent power law tail that describes fluctuations differing by as much as 8 orders of magnitude in this relative occurrence probability. The second empirical law concerns a histogram of size changes of business firms, which has a width that decreases as a power law of the firm size for firms that range over roughly 8 orders of magnitude. In addition to such scaling laws, there appears also the analog of "universality" - e.g., the analogous histogram of country size appears to obey the same scaling law, with the same exponent, as the histogram of firm size. (c) 1999 Elsevier Science B.V. All rights reserved.
\end{abstract}

\section{Introduction}

We begin by noting that the fundamental principles governing the complex system called economics are not completely uncovered. This observation seems to be

\footnotetext{
${ }^{*}$ Corresponding author. Fax: +1-617-3533783.

E-mail address: hes@bu.edu (H.E. Stanley)

0378-4371/99/\$ - see front matter (C) 1999 Elsevier Science B.V. All rights reserved. PII: S 0378-4371(99)00185-5
} 
almost generally accepted - e.g., the cover article of the 23 August 1997 issue of The Economist is entitled "The Puzzling Failure of Economics."

Then how can physicists contribute to the search for solutions to the puzzles posed by modern economics that economists themselves have not solved? One approach in the spirit of experimental physics - is to begin empirically, with real data that one can analyze in some detail, but without prior models. In economics, one has a great deal of real data. Moreover, if one has at one's disposal the tools of statistical physics and the computing power to carry out a number of approaches, this abundance of data is to great advantage. Thus, for physicists, studying the economy means studying a wealth of data on a well-defined complex system. Indeed, physicists in increasing numbers are finding problems posed by economics sufficiently challenging to engage their attention. Various terms have been applied to this new interdisciplinary subfield of physics. Some physicists prefer the term "Phynance". In an analogy with the terms biophysics, geophysics, and astrophysics, the term "Econophysics" was introduced in 1995 [1] to attempt to legitimize why physics graduate students obtaining physics Ph.D. degrees should be allowed to work on problems originating in economics.

If we physicists have any prior bias, it may be the lesson learned years ago when it was the fashion to work on critical phenomena: "Everything depends on everything else." A careful analysis of any system involves studying the propagation of correlations from one unit of the system to the next. We learned that these correlations propagate both directly and indirectly. At one time, it was imagined that "scale-free" phenomena are relevant to only a fairly narrow slice of physical phenomena [2]. However, the range of systems that apparently display power law and hence scale-invariant correlations has increased dramatically in recent years, ranging from base pair correlations in noncoding DNA [3] and their possible interpretation [4], lung inflation [5] and interbeat intervals of the human heart $[6,7]$ to complex systems involving large numbers of interacting units that display "free will," such as city growth [8,9], and even animal behavior $[10,11]$. In particular, economic time series, as e.g., stock market indices or currency exchange rates depend on the evolution of a large number of strongly interacting systems, and belong to the class of complex evolving systems. Thus, the statistical properties of financial markets have attracted the interests of many physicists [12-46]. In this short contribution, space limitations motivate us to focus mainly on our group's results; the work of other research groups is treated elsewhere. For a more balanced account, the reader should consult two recent books [47,48], other articles in these proceedings, and two other recent international conferences $[49,50]$.

\section{Correlations - or the Lack Thereof}

The recent availability of "high-frequency" data allows one to study economic time series on a wide range of time scales varying from seconds up to years. Consequently, a large number of methods known from statistical physics have been applied to characterize the time evolution of stock prices and foreign exchange rates [47]. 

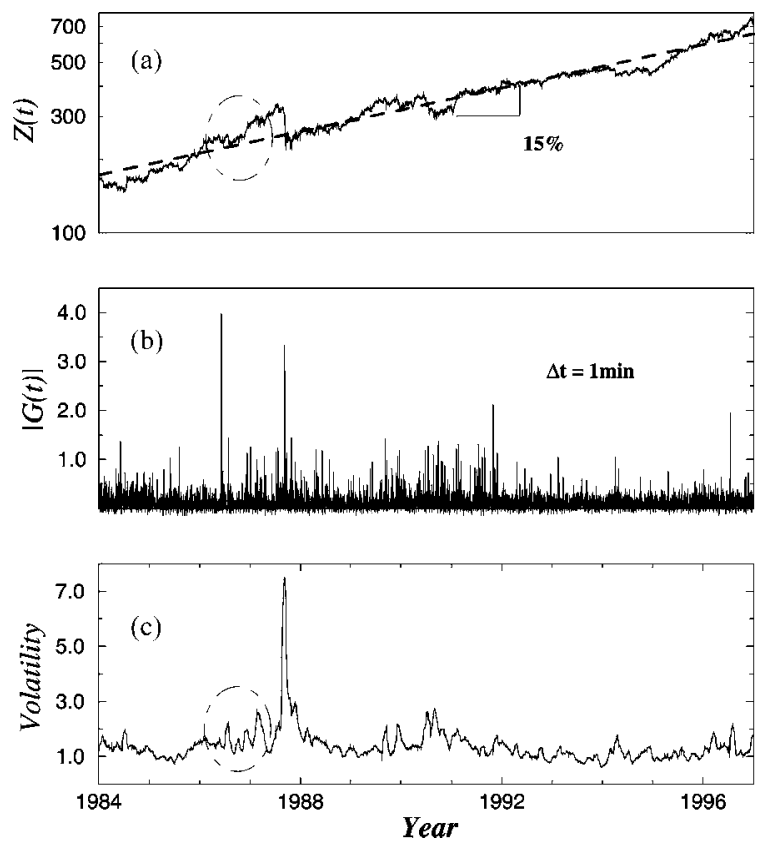

Fig. 1. (a) Data analyzed: The S\&P 500 index $Z(t)$ for the 13 -year period 3 January $1984-31$ December 1996 at sampling intervals $\Delta t=1 \mathrm{~min}$. These data include the data set analyzed by Mantegna and Stanley [12-14] and the extension of 7 extra years. Note the large fluctuations, such as that on 19 October 1987 ("black Monday"). The index $Z(t)$ has an increasing trend except for some crashes, such as the crashes in October 1987 and May 1990. For the period studied the index can apparently be fit by a straight line on a semi-log graph, i.e., exponential growth with annual increase rate of $\approx 15 \%$. (b) Amplitude of fluctuations, $|G(t)|$ (see text for definition), with $\Delta t=1 \mathrm{~min}$. (c) Volatility $V_{T}(t)$ with $T=1$ month $(8190 \mathrm{~min})$ and sampling time interval $\Delta t=30 \mathrm{~min}$ of the S\&P 500 index for the entire 13-year period 1984-1996. The highlighted block shows possible "precursors" of the October 1987 crash. This figure is provided courtesy of Y. Liu.

Much recent work is based on analysis of the S\&P 500 index, an index of the New York Stock Exchange that consists of 500 companies representative of the US economy. It is a market-value weighted index (stock price times number of shares outstanding), with each stock's weight in the index proportionate to its market value [12-14,51]. The S\&P 500 index is one of the most widely used benchmarks of US equity performance. Data that we analyzed typically cover a long period, such as 13 years (from January 1984 to December 1996) with a recording frequency of 1 min or shorter (Fig. 1a). The total number of data points in this 13-year period exceeds four million, more than three orders of magnitude greater than the classic Mandelbrot analysis of cotton price fluctuations [52].

The S\&P 500 index $Z(t)$ from 1984 to 1996 has an overall upward drift, approximately linear on a semi-log graph - interrupted by drastic events such as October 1987 and May 1990. Since the standard deviation of $Z(t+\Delta t)-Z(t)$ is proportional to the price level, one analyzes the logarithmic of the index changes $G(t) \equiv$ 

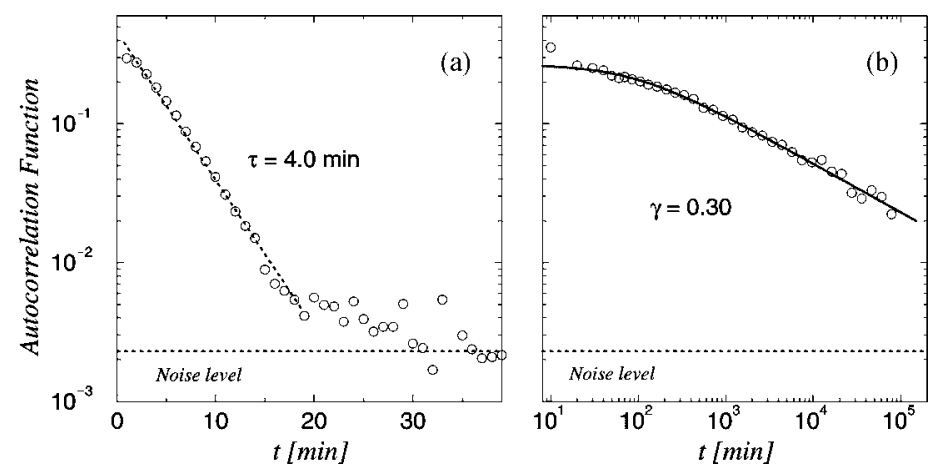

Fig. 2. (a) Semi-log plot of the autocorrelation function of $g(t)$. (b) Autocorrelation function of $|g(t)|$ in the double-log plot, with sampling time interval $\Delta t=1 \mathrm{~min}$. The horizontal dashed line indicates the noise level. The autocorrelation function of $g(t)$ decays exponentially to the noise level within half an hour, $C(t) \sim \exp (-t / \tau)$ with $\tau \approx 4.0 \mathrm{~min}$. In striking contrast, a power law correlation, $C(t) \sim t^{-\gamma}$, exists in the $|g(t)|$ for more than 3 decades. Note that both graphs are truncated at the first zero value of $C(t)$. The solid line in (b) is the fit to the function $1 /\left(1+t^{\gamma}\right)$ from which we obtain $\gamma=0.30 \pm 0.08$. This figure is provided courtesy of Y. Liu.

$\log _{e} Z(t+\Delta t)-\log _{e} Z(t)$, where $\Delta t$ is the time lag. One only counts the number of minutes during the opening hours of the stock market, and removes the nights, weekends and holidays from the data set, i.e., the closing and the next opening of the market is continuous.

It turns out that the distributions of the increments of economic time series, both in stock market indices and foreign currency exchange rates, are nearly symmetric and have very fat tails (strong "leptokurtic" wings) - see, e.g., [12-14,52-56]. Index increments as a function of time show exponentially decaying correlations that are at noise level after a few minutes (Fig. 2a) - making them fundamentally different from many well-studied examples of complex dynamical systems in physics such as, e.g., turbulent flow where power law correlations on long time scales are commonly observed [53-56].

The situation is different for the volatility, calculated, e.g., averaging market fluctuations over a suitable time interval (Fig. $1 \mathrm{~b}$ and 1c). There is long-time persistence in the volatility - much larger than the correlation time for price changes [51,57-61]. To quantify the dynamics of the volatility is important, since volatility is the key input of virtually all option-pricing models, including the classic Black and Scholes model [62] that is based on estimates of the asset's volatility over the remaining life of the option.

Specifically, using both traditional power spectrum methods as well as a new method - termed detrended fluctuation analysis (DFA) [63] - Liu, et al. [55] detect long-range volatility correlations embedded in a nonstationary time series, and avoid the spurious detection of apparent long-range correlations that are an artifact of nonstationarities (Fig. 2b).

To test whether this correlation is a spurious artifact of the distribution function, which might have long tails, Liu et al. shuffled each point of the volatility time series [51]. 


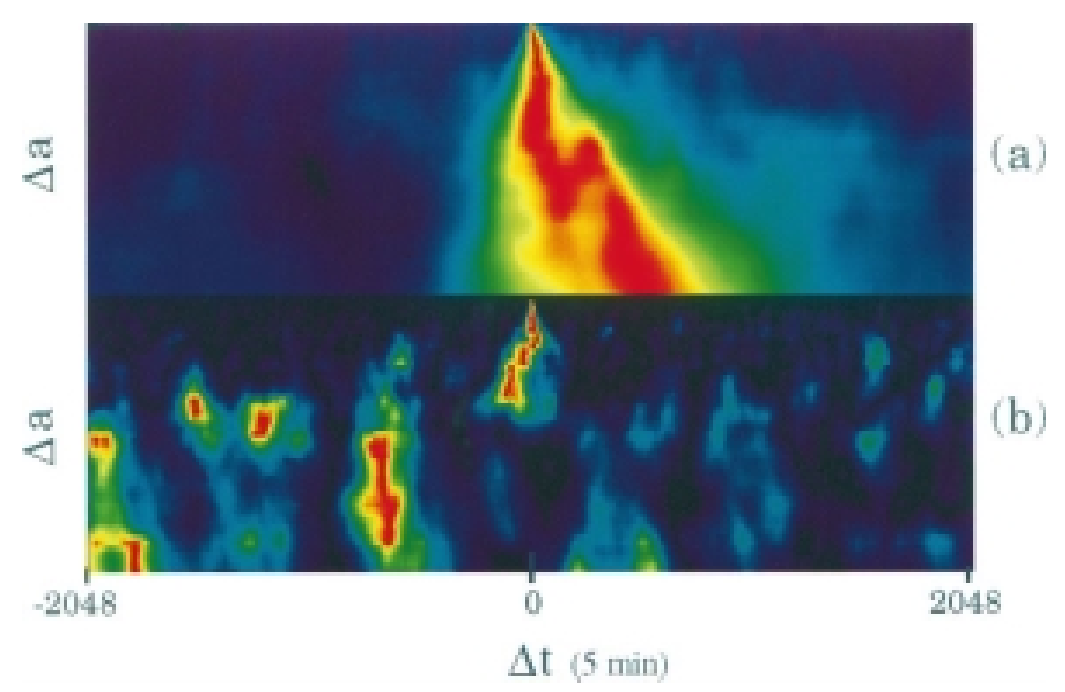

Fig. 3. The mutual information $I_{a}(\Delta t, \Delta a)$ of the variables $\left|G_{a}(t+\Delta t)\right|$ and $\left|G_{a+\Delta a}(t)\right|$ is represented in the ( $\Delta t, \Delta a$ ) half-plane ( 5 min units). Here $\Delta t$ is the time lag and $\Delta a$ is the time scale lag, and $\left|G_{a}(t+\Delta t)\right|$ is the price change over scale $a$ at time $t+\Delta t$. The time lag $\Delta t$ spans the interval [ - 2048,2048] while the scale lag $\Delta a$ ranges from $\Delta a=0$ (top) to 1024 (bottom). The amplitude of $I_{a}(\Delta t, \Delta a$ ) is coded from black for zero values to red for maximum positive values ("heat" code), independently at each scale lag $\Delta a$. (a) S\&P 500 index. (b) its randomly shuffled increment version. Note that, for middle scale lag values, the maxima (red spots) of the mutual information in (a) are 2 orders of magnitude larger than the corresponding maxima in (b). This figure is provided courtesy of A. Arneodo, M.-F. Muzy, and D. Sornette.

The random shuffling operation keeps the volatility distribution unchanged, but kills totally the correlations in the time series if there are any. DFA analysis of this randomly shuffled data does not show any correlations and gives exponent $\alpha=0.50$. This tells us that the long-range correlations are genuine and not a spurious artifact of the heavy-tailed distribution, because the distribution does not change when the data are shuffled.

Arneodo et al. have recently applied wavelet methods to decompose the volatility of intraday (S\&P 500) return data across scales [64]. They find a causal information cascade from large scales (i.e., small frequencies or "infrared") to fine scales ("ultraviolet"). Further, they devise a clever way to visualize the information flux across scales (see Fig. 3).

\section{Histograms of price changes}

As economic systems consist of a large number of interacting units, it is plausible that they might be amenable to scaling analysis. In fact, Mandelbrot in 1963 [52] demonstrated that the histogram of fluctuations in cotton prices obeys a scaling distribution, the Lévy distribution. A recent study determined that the high-frequency fluctuations in 
the S\&P 500 index also exhibits scaling behavior [12-14]. Analyzing almost one million records at $1 \mathrm{~min}$ intervals over 6 years of trading, Mantegna and Stanley determined that fluctuations on a 1-min time interval were reflected in 10-, 100- and 1000-min intervals [12-14]. The distribution of index returns fits a Lévy distribution with a sharp drop off in the tails. These scaling properties mean that viewing stock market returns on 1-min intervals provides insight on the behavior at 1000-min intervals.

Thus the Lévy part of the S\&P 500 distribution agrees with Mandelbrot's 1963 cotton price results, but the tail truncation does not (presumably because the tail statistics in the low-frequency results are not above the noise level). Recently, Gopikrishnan and co-workers have asked the question whether this discrepancy could arise from the fact that the S\&P is an average over many firms $[65,66]$. To this end, they analyze a database documenting each and every trade in the three major US stock markets, the New York Stock Exchange (NYSE), the American Stock Exchange (AMEX), and the National Association of Securities Dealers Automated Quotation (NASDAQ) for the entire 2-year period, January 1994 to December 1995. They thereby extract a sample of approximately $\approx 40$ million data points, which is much larger than the four million data points analyzed by Liu et al. [51,67,68], and the $\approx 2000$ data points studied by Mandelbrot. Gopikrishnan et al. find, remarkably, that an asymptotic power-law behavior, with an exponent $\alpha \approx 3$, for the cumulative distribution (Fig. 1 of Gopikrishnan et al. [65]) is well outside the Lévy regime $(0<\alpha<2)$. Similar values of $\alpha$ are found for the S\&P 500 index changes (Fig. 4). The volatility histogram can be analyzed in the same way as the price change histogram, with similar results (Fig. 5). It is interesting that the histogram of volatility changes was initially believed to be log-normal [51], but later work showed that the wings are by no means log-normal but are instead power law [68].

In summary, previous proposals for the histogram of index changes have included (i) a Gaussian distribution [69], (ii) a Lévy distribution [52,70,71], and (iii) a truncated Lévy distribution, where the tails become "approximately exponential" [12-14,72-76]. The inverse cubic result differs from all three proposals: Unlike (i) and (iii), it has diverging higher moments, and unlike (i) and (ii) it is not a stable distribution.

\section{Scale invariance in economics}

We and our collaborators have also studied economic data through those special eyeglasses that suggest that "everything depends on everything else." Specifically, in collaboration with a card-carrying economist, Michael Salinger - we studied the possibility that all the companies in a given economy might interact, more or less, like an Edwards-Anderson spin glass [77,78]. As in an Edwards-Anderson spin glass, each spin interacts with every other spin - but not with the same coupling and not even with the same sign.

If the sales of a given business firm A decrease by, e.g., $10 \%$, this will have repercussions in the economy. Some of the repercussions will be favorable - firm B, which 

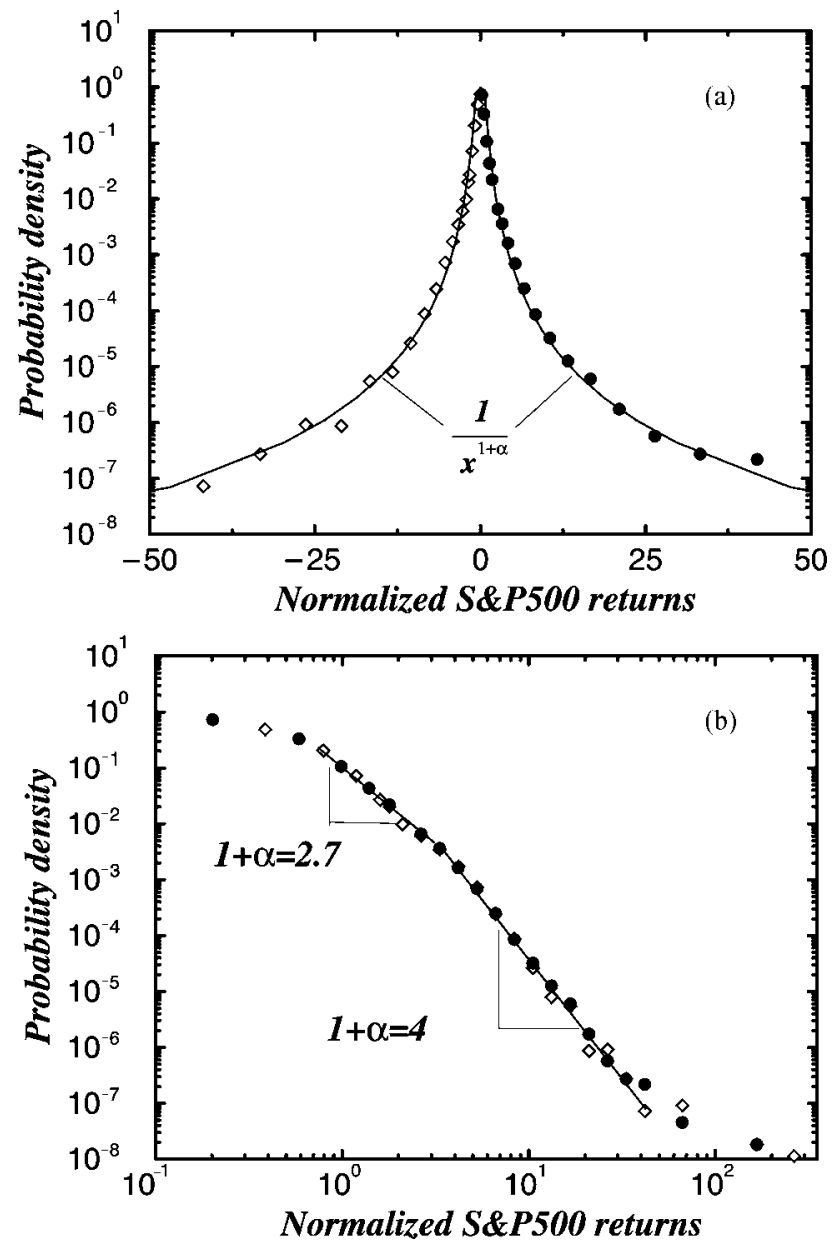

Fig. 4. (a) Probability distribution of the normalized price increments, where the abscissa is calculated in units of a standard deviation, for a database containing approximately 4 million records that lists at typically $15 \mathrm{sec}$ to $1 \mathrm{~min}$ intervals the value of the S\&P Index over the 13-year period January 1984 to December 1996. The lines are power-law fits to the data over the range from 2 to 80 standard deviations. (b) Log-log plot. The regression lines yield $\alpha \approx 3$. This figure is courtesy of P. Gopikrishnan, L.A.N. Amaral, and M. Meyer.

competes with A, may experience an increase in market share. Others will be negative - service industries that provide personal services for firm A employees may experience a drop-off in sales as employee salaries will surely decline. There are positive and negative correlations for almost any economic change. Can we view the economy as a complicated spin glass?

To approach this interesting bit of statistical "poetry" and make sense of it, M.H.R. Stanley and collaborators first located and secured a database - called COMPUSTAT - that lists the annual size of every firm in the United States. With this information, 

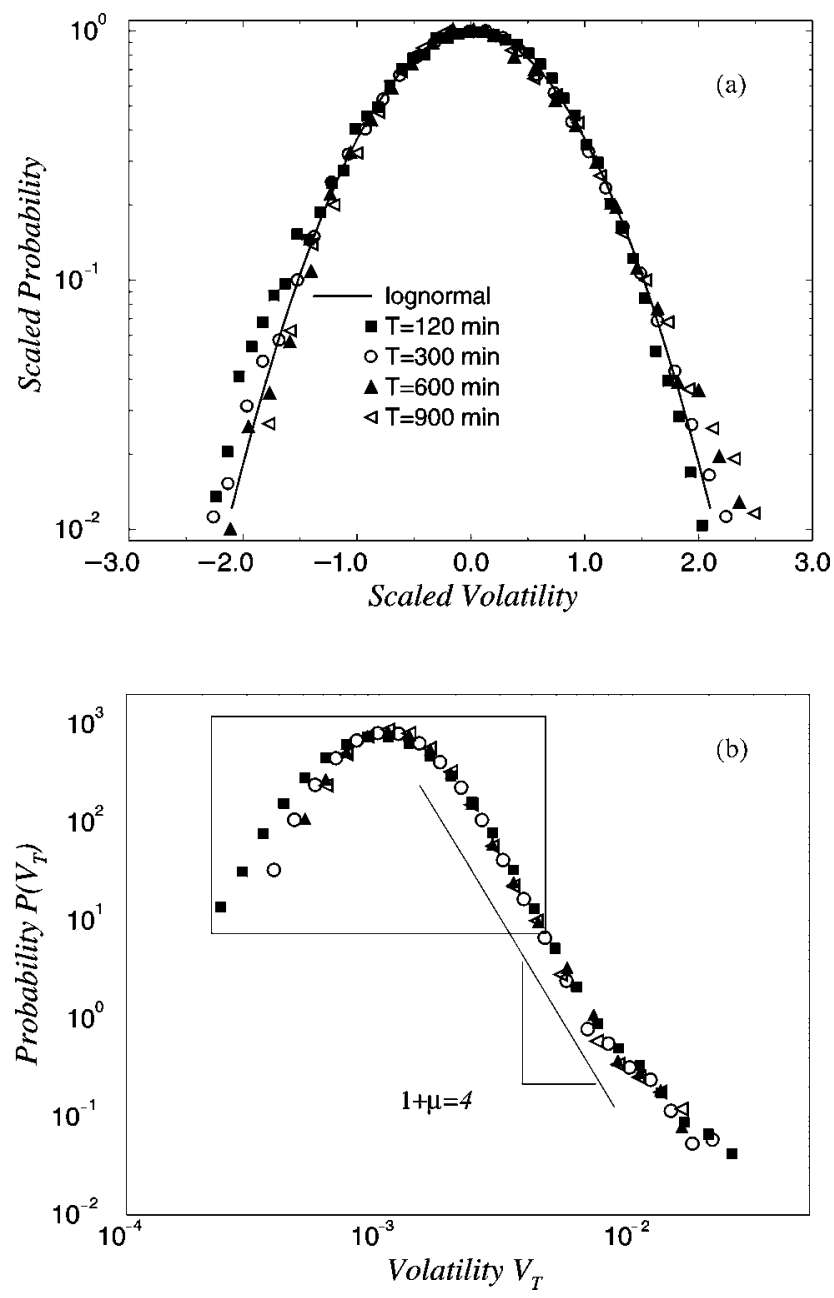

Fig. 5. Probability distribution of the volatility with different time windows $T$ with $\Delta t=30 \mathrm{~min}$. (a) The center part of the distribution shows a quadratic behavior on the log-linear scale. The asymptotic behavior seems consistent with a power law. The solid line is a fit to a long-normal distribution, using the log-normal scaling form, $\sqrt{v} \exp (a+v / 4) P\left(V_{T}\right)$ as a function of $\left(\ln V_{T}-a\right) / \sqrt{\pi v}$, where $a$ and $v$ are the mean and the width on a logarithmic scale. By the scaling, all curves collapse to the log-normal form with $a=0$ and $v=1, \exp \left(-(\ln x)^{2}\right)$ (solid line). (b) The full distribution (the box indicates the center of the distribution analyzed in (a)), now shown on a $\log -\log$ plot. It is apparent that the data fit well to a power-law with exponent four. The box shows the region of the distribution that appears to fit a log-normal distribution, and it is clear that the data even in this window are better fit by a power law. We hypothesize that other distributions that have been interpreted previously to be log-normal may in fact be power law distributions, since a log-normal distribution is almost indistinguishable from power-law distribution with a large value of the exponent. The figure is courtesy of Y. Liu. 
Stanley and co-workers calculated histograms of how firm sizes change from one year to the next [77,78]. They then made 15 histograms for each of 15 bins of firm sizes. The largest firms have very narrow distributions of growth rates - plausible because the percentage of size change from year to year for the largest firms cannot be that great. On the other hand, a tiny firm or a garage-based start-up can radically increase (or decrease) in size from year to year. Thus these 15 histograms have widths that depend on the firm size. When this width is plotted on the $y$-axis of $\log -\log$ paper as a function of firm size on the $x$-axis, the data are approximately linear over 8 orders of magnitude, from the tiniest firms in the database to the largest. The width scales as the firm size to an exponent $\beta$, with $\beta \approx 1 / 6[77,78]$. We can therefore normalize the growth rate and show that all the data collapse on a single curve - demonstrating the scaling of this measure of firm size.

Why does this data collapse occur? Researchers are working on that. Buldyrev models this firm structure as an approximate Cayley tree, in which each subunit of a firm reacts to its directives from above with a certain probability distribution [79]. More recently, Amaral et al. [80] have proposed a microscopic model that reproduces both the exponent and the distribution function. Takayasu and Okuyama [81] extended the empirical results to a wide range of countries, and developed still another model.

It is not impossible to imagine that there are some very general principles of complex organizations at work here, because similar empirical laws appear to hold for data on a range of systems that at first sight might not seem to be so closely related. For example, instead of studying the growth rate of firms, one can study the growth rates of countries by analyzing the ratio of the GDP of a country in one year compared to its value in the previous year. It appears (Fig. 6) that the histograms of country GDP sizes behave the same way as the histograms of firm sizes [82], even with the same value of the exponent $\beta \approx \frac{1}{6}$. Very recently, Plerou et al. analyzed in the same way a database comprising research budgets of 719 US universities and found similar qualitative results [83], but a larger exponent value, $\beta \approx \frac{1}{4}$. Amaral's model offers one possible explanation for this difference [80].

A surprising fact that may be related to the foregoing analysis is the following. Instead of the size of a firm at time $t$ (or the size of a GDP or a university budget) one might analyze the population $N_{s}(t)$ of a species $s$ in successive years. Such data exist for a 30-year period for every species sighted in North America, and very recently Keitt and Stanley $[11,84]$ have analyzed this database using the same sort of techniques used to describe long-term data sets on economics. They find statistical properties that are remarkably similar, and consistent with the idea that "every bird species interacts with every other bird species," just as the economic analysis supports the notion that "every economic entity interacts with every other economic entity."

These empirical results are not without interest, since they represent a departure from traditional models of economic systems - and bird populations - in which one partitions the entire data set into strongly interacting and weakly interacting subsets, and then oversimplifies or ignores the interactions in the weakly interacting subset. 

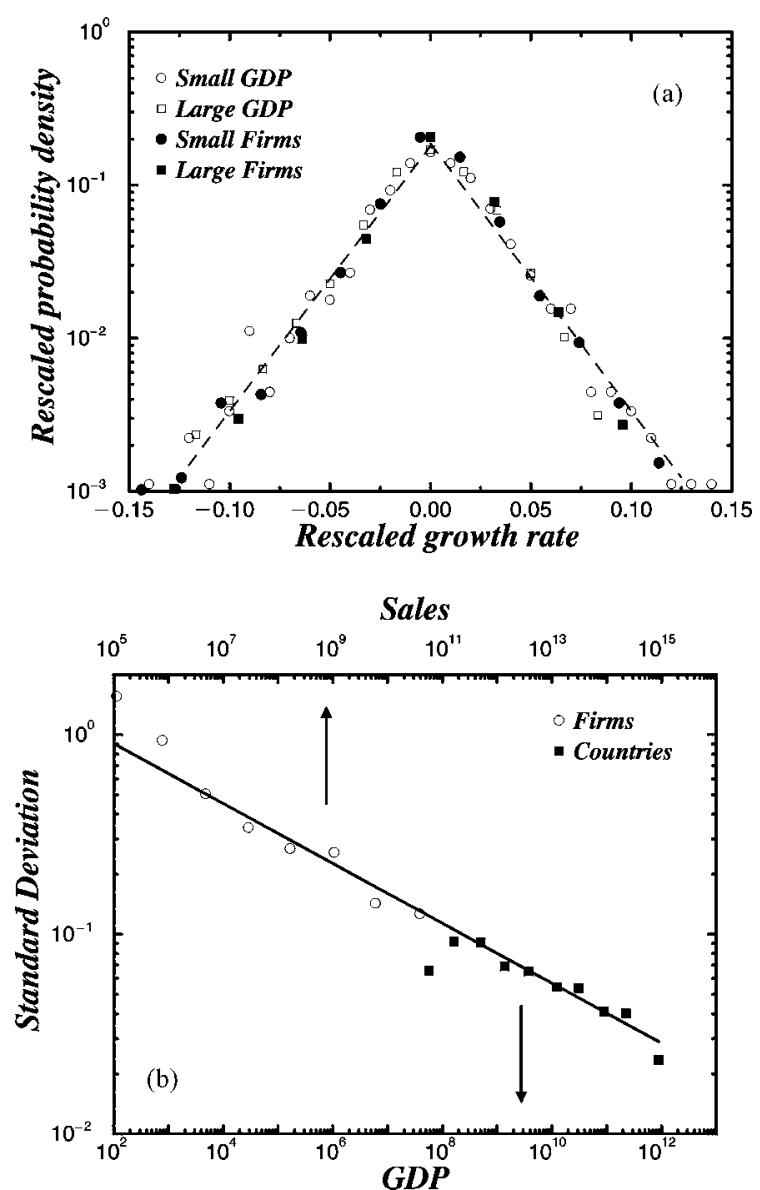

Fig. 6. Test of the similarity of the results for the growth of firms and countries. (a) Conditional probability density of annual growth rates for countries and firms. All rescaled data collapse onto a single curve showing that indeed the distributions have the same functional form. (b) Standard deviation of the distribution of annual growth rates. Note that the standard deviations decay with size with the same exponent for both firms and countries. The size is measured in sales for the firms (top axis) and in GDP for the countries (bottom axis). The firm data include all 4000 publicly traded manufacturing firms from the 19-year period 1974-1993 (see [77,78] for details), while the GDP data include 152 countries for the 43-year period 1950-1992 (see [82] for details). This figure is provided courtesy of Y. Lee, L.A.N. Amaral, D. Canning, and M. Meyer.

\section{Conclusions}

What can we say so far, other than just that apparently a number of natural questions in economics can be investigated quantitatively, using empirical analysis methods not unlike those used in the study of critical phenomena? And that the quantitative behavior of these complex economic systems - comprised of many animate units - is not unlike that found in interacting systems comprised of many inanimate submits. Can 
we understand why methods developed in, say, critical phenomena to quantify systems comprised of inanimate units should apparently apply to complex systems comprised of animate units? Indeed, the conceptual framework of critical phenomena is increasingly finding application in other fields, ranging from chemistry and biology [2] to econophysics [47,48] and even supercooled liquid water [85]. Why is this? One possible answer concerns the way in which correlations spread throughout a system, comprised of units in which "everything depends on everything else."

The paradox is simply stated: our intuition suggests that the correlation $C(r)$ between units separated by a distance $r$ should decay exponentially with $r$ - for the same reason the value of money stored in ones mattress decays exponentially with time (each year it loses a constant fraction of its worth - due to inflation). Thus we might expect that $C(r) \sim \mathrm{e}^{-r / \xi}$, where $\xi$, the correlation length, is the characteristic length scale above which the correlation function is negligibly small. Experiments and also calculations on mathematical models confirm that correlations usually do decay exponentially, but if the system is at its critical point, then the rapid exponential decay magically turns into a long-range power-law decay: magically $\xi \rightarrow \infty$.

So then how can correlations actually propagate an infinite distance, without requiring a series of amplification stations all along the way? We can understand such "infinite-range propagation" as arising from the huge multiplicity of interaction paths that connect two spins. The correlation between two spins along each of the interaction paths that connect them decreases exponentially with the length of the path. On the other hand, the number of such interaction paths increases exponentially, with a characteristic length that is temperature independent, depending primarily on the lattice dimension. This exponential increase is multiplied by a "gently decaying" power law that is negligible except for one special circumstance - the critical point. Right at the critical point, the gently decaying power-law correction factor in the number of interaction paths, normally negligible, emerges as the victor in this stand-off between the two warring exponential effects. As a result, two spins are well correlated even at arbitrarily large separation.

Will the power laws found empirically to describe complex economic systems ever be understood in analogous terms? Since we do not even know the nature of the interactions, if we consider 1000 firms then there are $1000 \times 999 / 2$ or half a million interactions to guess. What to do? The problem has recently been addressed in a novel way.

In some ways, the problem of interpreting the correlations between individual stockprice changes [86] is reminiscent of the difficulties experienced by physicists in the 1950s, in interpreting the spectra of complex nuclei. Large amounts of spectroscopic data on the energy levels were becoming available but were too complex to be explained by model calculations because the exact nature of the interactions were unknown. Random matrix theory was developed in this context, to deal with the statistics of energy levels of complex systems [87-94].

Recently, methods of random matrix theory have been applied to the analogous problem in finance [95-98]. For example, Plerou and her collaborators analyze the 
cross-correlation matrix of price changes at 30-min intervals of the largest 1000 US stocks for the 2-year period 1994-1995. In a strongly interacting system like the economy, one expects significant correlations between subunits. However, they surprisingly find that the statistics of most of the eigenvalues in the spectrum agree with the universal properties predicted by random matrix theory, but there are deviations only for a few of the largest eigenvalues. These deviations reflect cross-correlations between stocks not explainable purely by randomness.

Another genuine challenge is to achieve results that lead to respect from the economics community. Many in the economics community are receptive to new ideas and approaches of statistical physicists, and it is possible that if statistical physicists pay attention to the seminal work of economists, then the two fields can move forward in concert.

\section{Acknowledgements}

I conclude by thanking those under whose tutelage I learned what little I understand of this subject. In addition to those who consented to join me in co-authoring this brief report, these include the students, postdocs, and faculty visitors to our research group with whom I have enjoyed the pleasure of scientific collaboration. Those whose research provided the basis of this short report include: S.V. Buldyrev, P. Cizeau, S. Havlin, P.Ch. Ivanov, T.H. Keitt, H. Leschhorn, P. Maass, M. Meyer, C.-K. Peng, V. Plerou, M.A. Salinger, M.H.R. Stanley, and most especially R.N. Mantegna.

\section{References}

[1] H.E. Stanley, V. Afanasyev, L.A.N. Amaral, S.V. Buldyrev, A.L. Goldberger, S. Havlin, H. Leschhorn, P. Maass, R.N. Mantegna, C.-K. Peng, P.A. Prince, M.A. Salinger, M.H.R. Stanley, G.M. Viswanathan, Anomalous fluctuations in the dynamics of complex systems: from DNA and physiology to econophysics, Proceedings of 1995 Calcutta Conference on Dynamics of Complex Systems, Physica A 224 (1996) 302.

[2] C.M. Knobler, A. Robledo, H.E. Stanley, Statistical Mechanics in the Physical, Biological, and Social Sciences: Festschrift in Honor of Benjamin Widom on the occasion of his 70th Birthday, Elsevier, Amsterdam, 1997, Published as a special issue of Physica A 244 (1 Sept. 1997).

[3] C.-K. Peng, S.V. Buldyrev, A.L. Goldberger, S. Havlin, F. Sciortino, M. Simons, H.E. Stanley, Nature 356 (1992) 168.

[4] R.N. Mantegna, S.V. Buldyrev, A.L. Goldberger, S. Havlin, C.-K. Peng, M. Simons, H.E. Stanley, Phys. Rev. E 52 (1995) 2939.

[5] B. Suki, A.-L. Barabási, Z. Hantos, F. Peták, H.E. Stanley, Nature 368 (1994) 615.

[6] P.Ch. Ivanov, M.G. Rosenblum, C.-K. Peng, J. Mietus, S. Havlin, H.E. Stanley, A.L. Goldberger, Nature 383 (1996) 323.

[7] P.Ch. Ivanov, L.A.N. Amaral, A.L. Goldberger, S. Havlin, M.G. Rosenblum, Z. Struzik, H.E. Stanley, Multifractality in human heartbeat dynamics, Nature 399 (1999).

[8] H.A. Makse, S. Havlin, H.E. Stanley, Nature 377 (1995) 608.

[9] H.A. Makse, J. Andrade, M. Batty, S. Havlin, H.E. Stanley, Phys. Rev. E 58 (1998) 7054.

[10] G.M. Viswanathan, V. Afanasyev, S.V. Buldyrev, E.J. Murphy, P.A. Prince, H.E. Stanley, Nature 381 (1996) 413.

[11] T. Keitt, H.E. Stanley, Nature 393 (1998) 257. 
[12] R.N. Mantegna, H.E. Stanley, Nature 376 (1995) 46.

[13] A. Timmermann, Nature 376 (1995) 18.

[14] R.N. Mantegna, Physica A 179 (1991) 232.

[15] N. Vandewalle, M. Ausloos, Int. J. Mod. Phys. C 9 (1998) 711.

[16] N. Vandewalle, M. Ausloos, Eur. Phys. J. B 4 (1998) 257.

[17] N. Vandewalle, A. Boveroux, A. Minguet, M. Ausloos, Physica A 255 (1998) 201.

[18] T. Lux, M. Marchesi, Nature 297 (1999) 498.

[19] T. Lux, J. Econ. Behav. Organizat. 33 (1998) 143.

[20] T. Lux, J. Econ. Dyn. Control 22 (1997) 1.

[21] T. Lux, Appl. Econ. Lett. 3 (1996) 701.

[22] M. Levy, H. Levy, S. Solomon, Econ. Lett. 45 (1994) 103.

[23] M. Levy, S. Solomon, Int. J. Mod. Phys C 7 (1996) 65.

[24] J.-P. Bouchaud, R. Cont, Eur. Phys. J. B 6 (1998) 543.

[25] R. Cont, J.-P. Bouchaud, cond-mat/9712318.

[26] M. Potters, R. Cont, J.-P. Bouchaud, Europhys. Lett. 41 (1998) 239.

[27] L. Laloux, M. Potters, R. Cont, J.-P. Aguilar, J.-P. Bouchaud, cond-mat/9805212.

[28] J.-P. Bouchaud, D. Sornette, J. Phys. I (France) 4 (1994) 863.

[29] A. Johansen, D. Sornette, Int. J. Mod. Phys. C 10 (1999).

[30] D. Sornette, A. Johansen, J.-P. Bouchaud, J. Phys. I (France) 6 (1996) 167.

[31] D. Sornette, A. Johansen, Physica A 261 (1998) 581.

[32] D. Sornette, Physica A 256 (1998) 251.

[33] D. Stauffer, Ann. Phys.-Berlin 7 (1998) 529.

[34] D. Stauffer, T.J.P. Penna, Physica A 256 (1998) 284.

[35] D. Chowdhury, D. Stauffer, Eur. Phys. J. B 8 (1999) 477.

[36] I. Chang, D. Stauffer, Physica A 264 (1999) 1.

[37] D. Stauffer, T.J.P. Penna, Physica A 256 (1998) 284.

[38] D. Stauffer, P.M.C. de Oliveria, A.T. Bernardes, Int. J. Theor. Appl. Finance 2 (1999) 83.

[39] M. Marsili, Y.-C. Zhang, Phys. Rev. Lett. 80 (1998) 2741.

[40] G. Caldarelli, M. Marsili, Y.-C. Zhang, Europhys. Lett. 40 (1997) 479.

[41] S. Galluccio, G. Calderelli, M. Marsili, Y.-C. Zhang, Physica A 245 (1997) 423.

[42] M. Marsili, S. Maslov, Y.-C. Zhang, Physica A 253 (1998) 403.

[43] S. Maslov, Y.-C. Zhang, Physica 262 (1999) 232.

[44] D. Challet, Y.C. Zhang, Physica A 256 (1998) 514

[45] H. Takayasu, A.H. Sato, M. Takayasu, Phys. Rev. Lett. 79 (1997) 966.

[46] H. Takayasu, H. Miura, T. Hirabayashi, K. Hamada, Physica A 184 (1992) 127.

[47] J.-P. Bouchaud, M. Potters, Théorie des Risques Financiers: Portefeuilles, options et risques majeurs, Aléa, Saclay, 1997.

[48] R.N. Mantegna, H.E. Stanley, An Introduction to Econophysics: Correlations and Complexity in Finance, Cambridge University Press, Cambridge, 1999.

[49] J. Kertesz, I. Kondor (Eds.), Econophysics: Proceedings of the Budapest Workshop, Kluwer Academic Press, Dordrecht, 1999.

[50] K. Lauritsen, (Ed.), Application of Physics in Financial Analysis, Int. J. Theor. Appl. Finance [special issue] (1999).

[51] Y. Liu, P. Cizeau, M. Meyer, C.-K. Peng, H.E. Stanley, Physica A 245 (1997) 437.

[52] B.B. Mandelbrot, J. Business 36 (1963) 393.

[53] S. Ghashghaie, W. Breymann, J. Peinke, P. Talkner, Y. Dodge, Nature 381 (1996) 767.

[54] R.N. Mantegna, H.E. Stanley, Nature 383 (1996) 587.

[55] A. Arneodo, J.-P. Bouchaud, R. Cont, J.F. Muzy, M. Potters, D. Sornette, cond-mat/9607120.

[56] R.N. Mantegna, H.E. Stanley, Physica A 239 (1997) 255.

[57] Z. Ding, C.W.J. Granger, R.F. Engle, J. Empirical Finance 1 (1983) 83.

[58] M.M. Dacorogna, U.A. Muller, R.J. Nagler, R.B. Olsen, O.V. Pictet, J. Int. Money Finance 12 (1993) 413.

[59] R. Cont, Statistical finance: empirical study and theoretical modeling of price variations in financial markets, Ph.D. Thesis, Universite de Paris XI, 1998 (cond-mat/9705075).

[60] R. Cont, M. Potters, J.-P. Bouchaud, in: B. Dubrulle, F. Graner, D. Sornette (Eds), Scale Invariance and Beyond, Springer, Berlin, 1997. 
[61] M. Pasquini, M. Serva, cond-mat/9810232; cond-mat/9903334.

[62] F. Black, M. Scholes, J. Political Econ. 81 (1973) 637.

[63] C.-K. Peng, S.V. Buldyrev, S. Havlin, M. Simons, H.E. Stanley, A.L. Goldberger, Phys. Rev. E 49 (1994) 1684.

[64] A. Arneodo, J.-F. Muzy, D. Sornette, Eur. Phys. J. B 2 (1998) 277.

[65] P. Gopikrishnan, M. Meyer, L.A.N. Amaral, H.E. Stanley, Eur. J. Phys. B: Rapid Commun. 3 (1998) 139.

[66] P. Gopikrishnan, V. Plerou, L.A.N. Amaral, M. Meyer, H. Eugene Stanley, Scaling of the distributions of fluctuations in financial time series, Phys. Rev. E, submitted for publication. [cond-mat 9905305].

[67] P. Cizeau, Y. Liu, M. Meyer, C.-K. Peng, H.E. Stanley, Physica A 245 (1997) 441.

[68] Y. Liu, P. Gopikrishnan, P. Cizeau, M. Meyer, C.-K. Peng, H.E. Stanley, The statistical properties of the volatility of price fluctuations, Phys. Rev. E 60 (1999) [cond-mat/9903369].

[69] L. Bachelier, Ann. Sci. École Norm. Sup. 3 (1900) 21.

[70] V. Pareto, Cours d'Économie Politique, Lausanne, Paris, 1897.

[71] P. Lévy, Théorie de l'Addition des Variables Aléatoires, Gauthier-Villars, Paris, 1937.

[72] R.N. Mantegna, H.E. Stanley, Phys. Rev. Lett. 73 (1994) 2946.

[73] M.F. Shlesinger, Phys. Rev. Lett. 74 (1995) 4959.

[74] I. Koponen, Phys. Rev. E 52 (1995) 1197.

[75] A. Matacz, Financial modeling on option theory with the truncated Lévy process, Working Paper, School of Mathematics and Statistics, University of Sydney, Report 97-28, 1997.

[76] B. Podobnik, P. Ivanov, Y. Lee, H.E. Stanley, The exponentially-stretched truncated Levy flight, Phys. Rev. Lett., submitted for publication.

[77] M.H.R. Stanley, L.A.N. Amaral, S.V. Buldyrev, S. Havlin, H. Leschhorn, P. Maass, M.A. Salinger, H.E. Stanley, Nature 379 (1996) 804.

[78] L.A.N. Amaral, S.V. Buldyrev, S. Havlin, H. Leschhorn, P. Maass, M.A. Salinger, H.E. Stanley, M.H.R. Stanley, J. Phys. I France 7 (1997) 621.

[79] S.V. Buldyrev, L.A.N. Amaral, S. Havlin, H. Leschhorn, P. Maass, M.A. Salinger, H.E. Stanley, M.H.R. Stanley, J. Phys. I France 7 (1997) 635.

[80] L.A.N. Amaral, S.V. Buldyrev, S. Havlin, M.A. Salinger, H.E. Stanley, Phys. Rev. Lett. 80 (1998) 1385.

[81] H. Takayasu, K. Okuyama, Fractals 6 (1998) 67.

[82] Y. Lee, L.A.N. Amaral, D. Canning, M. Meyer, H.E. Stanley, Phys. Rev. Lett. 81 (1998) 3275.

[83] V. Plerou, L.A.N. Amaral, P. Gopikrishnan, M. Meyer, H.E. Stanley, Research at universities: ivory tower or competitive enterprise? Nature 400 (1999) [cond-mat/9811777].

[84] T. Keitt, H.E. Stanley, Lévy flights and $1 / f$ noise in high abundance populations, Proc. Nat. Acad. Sci., submitted for publication [cond-mat/981089].

[85] O. Mishima, H.E. Stanley, Nature 396 (1998) 329.

[86] R.N. Mantegna, cond-mat/9802256.

[87] E.P. Wigner, Ann. Math. 53 (1951) 36.

[88] E.P. Wigner, in: Conference on Neutron Physics by Time-of-flight, Gatdinburg, Tennessee, 1956.

[89] F.J. Dyson, J. Math. Phys. 3 (1962) 140.

[90] F.J. Dyson, M.L. Mehta, J. Math. Phys. 4 (1963) 701.

[91] M.L. Mehta, F.J. Dyson, J. Math. Phys. 4 (1963) 713.

[92] T. Guhr, A. Müller-Groeling, H.A. Weidenmüller, Phys. Rep. 299 (1998) 190.

[93] M.L. Mehta, Random Matrices, Academic Press, Boston, 1991.

[94] T.A. Brody et al., Rev. Mod. Phys 53 (1981) 385.

[95] L. Laloux, P. Cizeau, J.-P. Bouchaud, M. Potters, RISK Mag. (1999) 69.

[96] S. Galluccio, J.-P. Bouchaud, M. Potters, Physica A 259 (1998) 449.

[97] S. Galluccio, Y.C. Zhang, Phys. Rev. E 54 (1996) R4516.

[98] V. Plerou, P. Gopikrishnan, B. Rosenow, L.A.N. Amaral, H.E. Stanley, Universal and non-universal properties of cross-correlations in financial time series, Phys. Rev. Lett., submitted for publication, cond-mat/9903223. 